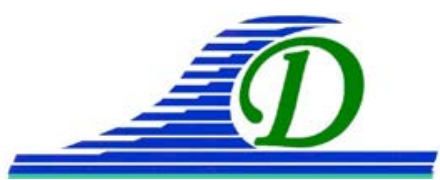

XIII ${ }^{\text {èmes }}$ Journées Nationales Génie Côtier - Génie Civil Dunkerque, 2-4 juillet 2014

DOI:10.5150/jngcgc.2014.073 C Editions Paralia CFL

disponible en ligne - http://www.paralia.fr - available online

\title{
Concevoir un rempiétement de quai
}

\section{Olivier MALASSINGNE ${ }^{1}$, Claire GALIANA ${ }^{2}$, Vianney CHANGENOT ${ }^{3}$, Nicolas ROUXEL ${ }^{1}$}

1. Centre d'Etudes et d'expertise sur les Risques, l'Environnement, la Mobilité et l’Aménagement - Direction Territoriale Ouest, Département Laboratoire de Saint Brieuc, 5 rue Jules Vallès, 22000 Saint Brieuc, France. olivier.malassingne@cerema.fr ; nicolas.rouxel@cerema.fr

2. Centre d'Etudes et d'expertise sur les Risques, l'Environnement, la Mobilité et l’Aménagement - Direction Territoriale Ouest, Département Infrastructure Mobilité Environnement Risques, MAN, 9 rue René Viviani, BP 46223, 44262 Nantes Cedex 2, France. claire.galiana@cerema.fr

3. Centre d'Etudes et d'expertise sur les Risques, l'Environnement, la Mobilité et l'Aménagement - Direction Technique Eau Mer et Fleuves, 134 rue de Beauvais, CS 60039, 60280 Margny Lès Compiègne, France. Vianney.changenot@cerema.fr

\section{Résumé :}

L'augmentation constante des caractéristiques des navires, et notamment de leur tirant d'eau pose souvent le problème du maintien des activités portuaires dans les bassins les plus anciens des ports. Lorsque le gestionnaire choisit d'approfondir le bassin existant, l'étude du rempiétement des quais est alors nécessaire. Le choix des techniques envisageables est conditionné par l'état du quai existant, son type (quai sur pieux, rideau de soutènement ou quai poids) et par la surface disponible du plan d'eau. Il est aussi à noter que la complexité du projet de rempiétement croît en fonction de l'interdépendance entre le nouvel ouvrage et l'ancien. Cet article présente les différents choix de rempiétement envisageable en fonction des différents types de quais et paramètres énoncés. Ces techniques sont détaillées dans une notice à paraître de la DTEMF du Cerema anciennement CETMEF.

Mots-clés : Travaux maritimes, Ouvrages portuaires, Rempiétement, Quai, Approfondissement, Souille.

\section{Introduction}

L'augmentation constante des caractéristiques des navires de commerce, de croisières et de pêche voire de loisirs, en particulier l'accroissement de leur tirant d'eau ainsi que des sollicitations des engins d'exploitation portuaire pose souvent le problème du maintien des activités portuaires dans les bassins les plus anciens des ports. 


\section{Thème 4 - Ouvrages portuaires et offshore}

Pour répondre à cette évolution, les gestionnaires portuaires se trouvent dans l'obligation d'investir :

a) soit dans la construction de nouveaux quais,

b) soit dans l'adaptation de quais anciens.

Dans ce dernier cas l'approfondissement des bassins nécessite généralement l'étude du rempiètement des quais. Cet article présente les différentes techniques envisageables en fonction des différents types de quais mais aussi des objectifs et usages envisagés et en fonction des capacités d'évolutions du plan d'eau et de la place disponible.

Les quais peuvent être classés en 3 grands types en fonction de leur mode construction et surtout selon les principes de calculs qui les régissent :

a) les quais sur pieux ;

b) les rideaux de soutènements formant un soutènement plan du sol présentant généralement des ancrages de tirant actifs ou passifs, avec ou sans contre-rideaux. Cette dénomination regroupe les ouvrages réalisés en palplanches, en berlinoise, et en parois moulées etc. ;

c) les quais poids. Ce dernier cas regroupe les quais de type mur en maçonnerie, en béton ou caissons. Pour ce type de quai il est à noter qu'il existe une grande variété de techniques offertes pour le rempiètement.

Il est aussi à remarquer que la complexité du projet de rempiètement croît en fonction de l'interdépendance entre le nouvel ouvrage et l'ancien.

Cet article présente les différents choix de rempiétement envisageable en fonction des différents types de quais et différents paramètres à prendre en compte. Ces techniques sont détaillées dans une notice à paraître de la DTEMF du Cerema anciennement CETMEF.

\section{Les pré-requis à une étude de rempiétement}

Un rempiétement est la reprise en sous-œuvre des fondations d'un mur, d'un bâtiment. Dans le cas de quais, ce terme est employé pour désigner la reprise en sous-œuvre ou bien le confortement d'un quai existant dans le cas d'un approfondissement du bassin. Par extension, le rempiétement désigne aussi certaines modifications de quais par création d'ouvrages neufs au-devant des premiers en interaction ou liaisonnés aux premiers. Les rempiètements peuvent être classés selon deux modes : soit en fonction de l'interaction de la nouvelle structure avec l'ancien quai, soit en fonction du type de quai existant. Le choix du second mode a été effectué dans cet article.

Lorsqu'un gestionnaire envisage la réalisation d'un rempiètement, il doit essayer de répondre aux questions suivantes :

a) Quel est le type du quai existant ? Comment fonctionne-t-il mécaniquement ?

b) Quel est l'état de la structure existante ?

c) Quelle est la superficie de mon plan d'eau (possibilité de réaliser une structure éloignée du quai existant ou non? 


\section{XIII ${ }^{\text {èmes }}$ Journées Nationales Génie Côtier - Génie Civil \\ Dunkerque, 2-4 juillet 2014}

d) Quel est la nature du sol, quels types de nouvelles structures conditionne-t-elle ?

e) Quel est l'historique complet du quai existant, en particulier lors de sa construction, ainsi que sa géométrie?

Les réponses à ces questions permettent en effet de déterminer les différentes solutions possibles de rempiètement et conditionnent aussi les études à entreprendre. Le type de quai existant induit aussi les possibilités de rempiètement envisageable. Seuls les quais poids en maçonnerie connaissent peu de restrictions. Un diagnostic détaillé permet de connaître l'état du quai ou de la structure existants. Dans le cas où le quai est en bon état, sa réutilisation en interdépendance avec le rempiètement est envisageable. Il convient de vérifier si la structure ancienne peut supporter une augmentation des sollicitations par des essais complémentaires, géotechniques et/ou de résistance des matériaux, et aussi d'effectuer un nouveau calcul de l'ouvrage. Si des défaillances importantes sont relevées, la réalisation d'un rempiètement indépendant ou peu dépendant avec le quai existant est préférable. Certains systèmes constructifs permettent également de conforter l'ouvrage existant tout en permettant un approfondissement de la souille au devant (cas de réalisation de parois de soutènement par exemple).

La surface disponible du bassin a aussi un impact sur le choix du type ou de la gamme de rempiètement possible en fonction de la profondeur et du type de bateau accueilli.

La nature du sol sous l'ouvrage conditionne ou favorise certains types de rempiètement. Dans des cas extrêmes de sols résistants (rocheux), un terrassement seul peut satisfaire aux exigences d'approfondissement du bassin.

La connaissance de la géométrie de l'ouvrage existant, ainsi que les méthodes et phasages de constructions de l'ouvrage ancien est primordiale voir conditionne la réalisation de certains rempiètements. En effet le recueil de ces données est impératif dans le cas de calculs de déplacements aux éléments finis, calculs généralement réalisés pour les rempiètements interdépendant des anciennes structures.. Ce recueil peut alors entrainer des études approfondies et conséquentes pour son obtention en l'absence de dossier d'ouvrage ou d'archives suffisamment précises.

Au préalable de chaque étude de projet de rempiètement, il convient de rechercher les paramètres géotechniques et caractéristiques mécaniques des sols en place et des remblais adjacents ainsi que les différents niveaux piézométriques. La recherche de polluants (métaux lourds dans les sédiments ...) sera aussi nécessaire. Car en cas de présence avérée, les coûts de dragage ou de déchargement du quai peuvent alors fortement augmenter, ce qui se répercute sur le coût du projet ou bien conduit à choisir d'autres techniques de rempiètement. 


\section{Les différentes typologies de quais, les différents types de rempiètement et leurs impacts}

\subsection{Les quais sur pieux}

Ces quais sont constitués d'une plateforme supportée par des pieux et présentent un raccordement de la souille au terre-plein par un talus et/ou un soutènement.

La fonction de soutènement, propre aux quais sur pieux, est assurée par la combinaison d'un talus et d'un rideau-plan ou voile placé en arrière ou en avant du quai, avec ou sans tirants d'ancrage. Le voile arrière peut également être conçu pour participer à la reprise des efforts horizontaux. En dehors de la création d'un nouvel ouvrage devant le premier ou un approfondissement de la souille en dehors de la zone d'influence des fondations, il existe qu'une seule possibilité d'amélioration des capacités de ce type de quai par réalisation d'un écran de soutènement en pied du talus.

Cet écran est généralement réalisé Lorsque l'emprise du plan d'eau est réduite au moyen de palplanches et pieux métalliques ou d'une paroi berlinoise. Une légère augmentation de la plateforme est néanmoins nécessaire afin d'ancrer les palplanches dans une nouvelle poutre de couronnement. Il est à noter que les efforts horizontaux générés par la poussée des terres sur l'écran peut nécessiter la réalisation d'ancrages de type tirants ou de pieux inclinés dans le terre-plein.

\subsection{Ecrans de soutènements}

D'une manière générale, une augmentation de la profondeur de la souille devant une paroi est possible. La diminution de la fiche dans le sol entraîne une augmentation des efforts, de flexion notamment dans l'écran. Dans le cas d'ancrages, ceux-ci sont aussi fortement sollicités. Lorsque le voile est encastré en pied, l'approfondissement de la souille entraîne une augmentation du moment maximal, les efforts d'encastrement peuvent alors être remplacés par une simple butée. La réalisation de nouveaux ancrages est souvent nécessaire afin d'assurer la stabilité de la paroi. Il est aussi à noter une augmentation des gradients hydrauliques pouvant générer des renards notamment dans le cas de plateformes constituées de matériaux fins. Ce point doit être vérifié par des calculs hydrauliques.

Il est aussi possible de diminuer la poussée des terres sur l'écran (déchargement) d'une des manières suivantes :

a) par réalisation d'un terrassement à l'arrière de l'écran et édification d'une plate-forme sur pieux ou sur sols mixés ou inclusions rigides

b) par remplacement des matériaux tout-venant du terre-plein par des matériaux plus frottants.

Ces solutions nécessitent de connaître, voire de mettre à jour les ancrages afin de ne pas les endommager lors de la réalisation des pieux ou des terrassements, si leur conservation est nécessaire. 


\section{XIII ${ }^{\text {èmes }}$ Journées Nationales Génie Côtier - Génie Civil \\ Dunkerque, 2-4 juillet 2014}

Pour les écrans de type rideau de palplanches métalliques, il est toujours possible de renforcer la structure et de réaliser de nouveaux tirants d'ancrages. Les seules limites sont l'état de la structure vis-à-vis de la corrosion, l'épaisseur sacrificielle résiduelle, la longueur du voile et les possibilités de réalisation du nouveau lit de tirants. Pour ce type d'écran, lorsque le plan d'eau le permet, il est toujours possible de réaliser un nouveau soutènement en avant du premier et de se servir de l'ancien écran comme d'un rideau d'ancrage arrière (contre rideau) pour y sceller les nouveaux tirants d'ancrages. Cependant la réalisation d'un second rideau de palplanches accolé au premier est plus fréquente.

Les parois moulées sont actuellement avec les quais sur pieux, une technique de réalisation des quais permettant d'obtenir les plus forts tirants d'eau, de l'ordre d'une vingtaine de mètres, parfois plus. De part leur conception récente et les grandes possibilités qu'ont offertes cette technique, il semble qu'il y ait peu d'exemples de rempiètement de quai en parois moulées. De plus compte tenu du ferraillage important des parois, il est délicat de pouvoir réaliser de nouveaux tirants d'ancrage sans endommager les armatures. Il faut également vérifier que le ferraillage puisse reprendre les nouveaux efforts. La seule possibilité offerte semble être la réalisation d'un nouveau quai sur pieux en avant du soutènement.

\subsection{Murs poids}

Cette dénomination regroupe différents types de quais dont la stabilité est assurée par le poids de l'ouvrage lui-même. Ces quais massifs comprennent l'ensemble des quais avec des murs en maçonnerie, la plupart des quais en béton ou les quais caissons. Ces quais présentent généralement des hauteurs inférieures aux types de quais précédents (paroi ou sur pieux) et de ce fait ont fait et font souvent l'objet de rempiètement. Ils sont aussi de conception plus ancienne que les types de quais précédents.

Compte tenu de leur principe de fonctionnement, ces quais, à l'exception des quais caissons, présentent un fruit vers l'amont pour la paroi visible et des redans à l'arrière. Il sont donc étroits en tête et larges à leur base.

La réalisation de structures flottantes devant les quais existants, bien qu'il ne s'agisse pas de solutions de rempiétement à proprement parler, est généralement la solution effectuée à grande échelle pour l'édification de ports de plaisance ou de pèche afin d'accueillir des embarcations de taille petite à moyenne. Cette solution est avantageuse car elle permet d'augmenter rapidement le linéaire de front d'accostage en réalisant des cheminements flottants ou catway à moindre coût.

Comme pour les autres types de quais, il est toujours possible d'approfondir la souille en dehors de la zone d'influence des fondations et de créer ainsi un nouveau talus en avant du quai. La pente du nouveau talus en avant du quai est déterminée en fonction de la nature du sol et des résultats de calculs de la stabilité au grand glissement du projet envisagé. Il est donc nécessaire de connaître la géométrie exacte du quai (largeur, 


\section{Thème 4 - Ouvrages portuaires et offshore}

profondeur, nombre de redans...) ce qui entraîne généralement sur les quais anciens des études géotechniques importantes si les plans d'origine n'ont pas été conservés ou pour vérification de ces derniers. Dans le cas où le quai est fondé sur du rocher présentant de bonnes caractéristiques mécaniques, le talus peut alors être vertical à sub-vertical en pied d'ouvrage.

La réalisation d'un rempiétement par création d'un quai sur pieux au devant d'un mur poids peut être envisagée si la surface du plan d'eau est suffisante pour accepter ce type de structure. Afin d'optimiser la réalisation, la nouvelle structure doit s'accompagner d'un approfondissement de la souille si possible en dehors de la zone d'influence des fondations du quai poids par création d'un nouveau talus en avant du quai. Il convient de vérifier la stabilité au grand glissement du projet envisagé afin de dimensionner au mieux la pente du talus qui varie en fonction de la nature du sol, et le cas échéant la stabilité seule de l'ancien quai dans le cas de terrassements dans sa zone d'influence.

En fonction de l'état du quai et du projet, le mur poids peut servir de support ou d'ancrage arrière pour le nouveau quai sur pieux. Ce type de réalisation peut aussi être envisagé pour ne pas interférer avec l'ancien quai du fait d'un degré d’altération avancé ou par absence de connaissance sur sa géométrie exacte. Dans ce cas la réalisation du quai sur pieux s'apparente à la création d'un ouvrage neuf. Afin d'offrir un approfondissement plus important de la souille, un soutènement peut être associé au quai sur pieux.
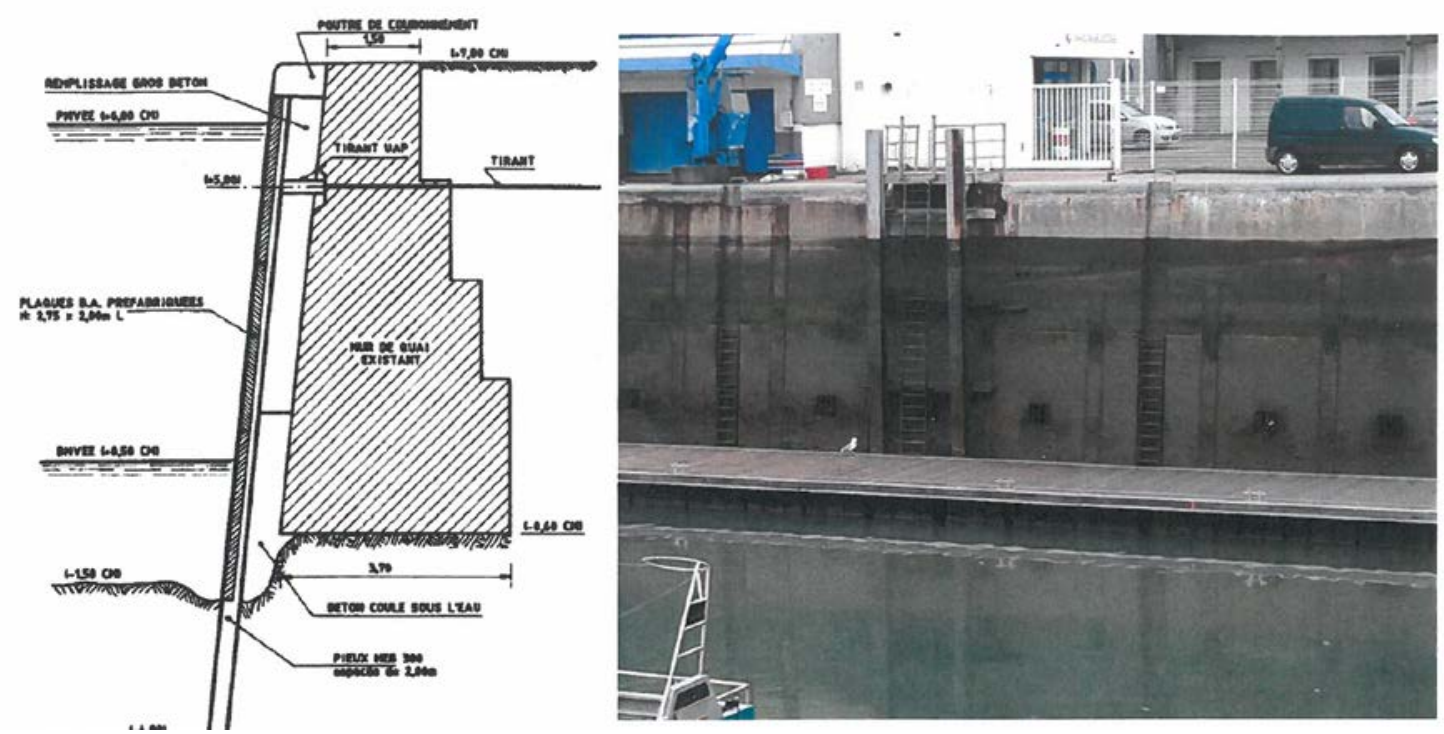

Figure 1. Rempiètement par création d'une paroi berlinoise, quai de la criée des Sables d'Olonne avec coupe transversale extraite de la notice Cetmef PM 94.02 fascicule 1.

La réalisation d'un soutènement complet devant l'ouvrage s'avère nécessaire lorsque la surface du plan d'eau est restreinte et que l'ancien quai pourrait difficilement supporter 


\section{XIII ${ }^{\text {èmes }}$ Journées Nationales Génie Côtier - Génie Civil \\ Dunkerque, 2-4 juillet 2014}

les nouvelles charges d'exploitation, notamment en terme d'efforts d'accostage. Ce type d'ouvrage est aussi effectué lorsque les quais anciens en maçonnerie présentent des désordres importants (lacunes ou autre) nécessitant des travaux de réparation conséquents. Le rempiètement ainsi créé permet à la fois de conforter le quai mais aussi d'en augmenter les capacités.

En fonction de la place disponible, de la profondeur du rempiètement et de l'état de l'ancien quai, le nouveau soutènement est soit interdépendant de l'ancien quai, soit les ancrages de la paroi sont liaisonnés avec le quai en maçonnerie, afin de servir de contrerideau. Le nouveau soutènement est réalisé soit à l'aide de palplanches métalliques, soit à l'aide d'une paroi berlinoise (pieux métalliques et plaques béton préfabriquées).

La création d'un nouveau soutènement uniquement au pied du quai existant nécessite que les conditions suivantes soit réunies (totalement ou partiellement) :

a) l'ouvrage ancien est en bon état et ne nécessite pas de réparations importantes,

b) le quai ne recevra pas de sollicitations supérieures à celles qu'il reçoit déjà,

c) la géométrie de l'ancien quai est connue,

d) le plan d'eau est restreint.

En fonction de la position du soutènement, deux cas mécaniquement différents, peuvent être distingués :

a) soit le nouveau soutènement est indépendant du quai poids

b) soit le soutènement est liaisonné à l'ancien quai par la réalisation d'une lierne et /ou d'ancrages en tête du rideau.

Il est à noter que les calculs de dimensionnement de ce type de réalisation sont complexes. Les recours à des modèles par éléments finis sont souvent nécessaires. Cette modélisation nécessite de connaître l'intégralité des contraintes que les sols ont subit et donc de modéliser la réalisation du quai existant, phases provisoires y compris, avant de pouvoir étudier le nouveau projet.

Diverses techniques d'amélioration de sols peuvent être utilisées dans le cadre de rempiétements. Elles se caractérisent par l'adjonction d'un liant hydraulique aux sols naturels ou aux remblais situés en arrière du mur de quai par des machines de forage, de réalisation de pieux ou de barrettes. En fonction de cette nature de sol, il est possible de réaliser soit de l'injection (sols sableux de préférence, l'intégralité du massif est injectée) soit du Jet Grouting : réalisation de colonnes de sols mixés avec un liant par un forage sous haute pression soit d'inclusions rigide de type colonne à module contrôlée (sol mixé à la tarière creuse) dans les remblais de quais. Le but recherché est l'amélioration des caractéristiques mécaniques, de la cohésion et de l'angle de frottement en particulier, afin d'augmenter la résistance au cisaillement des sols ou remblais environnant. 


\section{Conclusions}

Bien que de multiples techniques soient disponibles pour rempiéter un quai, les exemples récents semblent rares, malgré une enquête approfondie auprès des services gestionnaires. Les techniques les plus couramment observées en rempiètement sont actuellement la réalisation :

a) d'un soutènement en pied de talus pour les ouvrages sur pieux

b) d'ancrages supplémentaires pour les écrans de soutènement

c) d'un soutènement en pied d'ouvrage pour les quais poids en bon état ou par paroi berlinoise dans le cas de quais poids dégradés.

Cependant il convient de noter que des généralités sont délicates à établir tant l'étendue des techniques possibles, en particulier pour les quais poids est importante. Chaque technique ayant ses avantages et inconvénients, une étude au cas par cas, par un spécialiste, permet de valider le ou les types de rempiètement optimal.

\section{Bibliographie}

ARISTAGHES P. (1990). Calcul des rempiètements de quai. Annales des Ponts et Chaussées, 1er-2è trimestre 1990, pp 37-49.

BOUGNOL J. Notice de rempiétement de quai Document provisoire STCPMVN inédit. CETMEF (1994). Notice PM 94.02, Auscultation, surveillance et entretien des ouvrages maritimes, Fascicule 1 : les ouvrages en maçonnerie. CETMEF, 86 p.

CETMEF (2002). Notice PM 02.02. Surveillance, auscultation et entretien des ouvrages maritimes, Fascicule 5 : Quais en parois moulées. CETMEF, 86 p.

DUPLAINE H., GARRISSOU J., COUTURES J.Y. (2003). Modernisation des postes 415 et 416 au Port Autonome de Bordeaux Bassens. Revue Travaux n 797, pp 71-75.

GOUVENOT D., DE BUYSERE G. (1980). Les fondations spéciales en travaux maritimes. Revue Excavator 2/80, pp 22-42.

HOUY A. (1981). Les palplanches en acier dans les Ouvrages Maritimes. Revue Excavator 9/81, pp 25-45.

LE GORGEU V., GUITONNEAU R. (1954). Reconstruction de la digue de l'Est à Dunkerque. Proceedings of 5th Conference on Coastal Engineering, Grenoble, France, Chapter 38, pp 555-586

LEVILLAIN J.P. (1992). Rempiètement des murs de quai : quelques techniques. Journées Nationales Génie Côtier - Génie Civil, Nantes, pp 236-260. http://dx.doi.org/10.5150/jngcgc.1992.017-L

MESTAT P., HUMBERT P., DUBOUCHET A. (2000). Recommandations pour la vérification de modèles d'éléments finis en géotechnique. Bulletin des Laboratoires des Ponts et Chaussées n²29, pp 33-51.

ROUXEL N. (2007). Suivi du confortement du quai Carnot à Concarneau. Club Ouvrages Maritimes. CETE OUEST. Disponible en ligne sur : http://www.cete-ouest.developpementdurable.gouv.fr/IMG/pdf/fiche_Carnot_v2-1_cle2d7a81.pdf 